\title{
Using Harmonic Mean to Solve Multi-Objective Linear Programming Problems
}

\author{
Nejmaddin A. Sulaiman, Rebaz B. Mustafa* \\ Department of Mathematics, College of Education, University of Salahaddin, Erbil, Iraq \\ Email: "bahramrebaz@yahoo.com
}

Received 11 November 2015; accepted 15 January 2016; published 20 January 2016

Copyright (C) 2016 by authors and Scientific Research Publishing Inc.

This work is licensed under the Creative Commons Attribution International License (CC BY).

http://creativecommons.org/licenses/by/4.0/

c) (i) Open Access

\begin{abstract}
In this paper, we have suggested a new technique to transform multi-objective linear programming problem (MOLPP) to the single objective linear programming problem by using Harmonic mean for values of function and an algorithm is suggested for its solution, the computer application of algorithm has been demonstrated by solving some numerical examples. We have used some other techniques, such as (sen, arithmetic mean, median) to solve the same problems, the results in Table 3 indicate that the new technique in general is promising.
\end{abstract}

\section{Keywords}

\section{MOLPP, Harmonic Mean}

\section{Introduction}

Linear programming is a relatively new mathematical discipline, dating from the invention of the simplex method by G. B. Dantzig in 1947. He proposed the simplex algorithm as an efficient method to solve a linear programming problem.

A multi-objective linear programming problem is introduced by Chandra Sen [1] and suggests an approach to construct the multi-objective function under the limitation that the optimum value of individual problem was greater than zero. [2] studied the multi-objective function by solving the multi-objective programming problem, using mean and mean value. [3] solved the multi objective fractional programming problem by Chandra Sen's technique. In order to extend this work, we have defined a multi-objective linear programming problem and in-

${ }^{*}$ Corresponding author. 
vestigated the algorithm to solve linear programming problem for multi-objective functions. By new technique, we use harmonic mean (HM) of the values of objective functions. The computer application of our algorithm has also been discussed by solving some numerical examples. Finally we have showed results and comparison among the new technique and Chandra Sen’s approach [1] and Sulaiman's approach [2].

\section{Mathematical Definition of Multi-Objective Programming Problems (MOPP)}

A deterministic (MOPP) model is usually formulated to maximize and/or minimize several objectives simultaneously subject to a constraint set with " $\geq$ ” and/or " $\leq$ ” relationships the equality constraints may be expressed as a combination of both of inequality constraints.

Mathematically, the MOPP problems can be defined as:

$$
\left.\begin{array}{ll}
\text { Max. } f_{i}=c_{i} X+\alpha_{i} & i=1, \cdots, r \\
\text { Min. } f_{i}=c_{i} X+\alpha_{i} & i=r+1, \cdots, s
\end{array}\right\}
$$

subject to:

$$
\begin{aligned}
& A X\left[\begin{array}{l}
\geq \\
= \\
\leq
\end{array}\right] B \\
& X \geq 0
\end{aligned}
$$

where $x$ is an n-dimensional vector of decision variables $c$ is n-dimensional vector of constants, $B$ is $\mathrm{m}$-dimensional vector of constants, $r$ is the number of objective function to be maximized, $s$ the number of objective function to maximized plus minimized, $(s-r)$ is the number of objective that is to be minimized, $A$ is a $(m \times n)$ matrix of coefficients all vectors are assumed to be column vectors unless transposed, $\alpha_{i}(i=1, \cdots, s)$ are scalar constants, $c_{i} X+\alpha_{i}(i=1, \cdots, s)$ are linear factors for all feasible solutions [3].

If $\alpha_{i}=0$; for all $i=1, \cdots, s$, then the mathematical form become:

$$
\left.\begin{array}{ll}
\text { Max. } f_{i}=c_{i} X+\alpha_{i} & i=1, \cdots, r \\
\text { Min. } f_{i}=c_{i} X+\alpha_{i} & i=r+1, \cdots, s
\end{array}\right\}
$$

subject to:

$$
\begin{aligned}
& A X=B \\
& X \geq 0
\end{aligned}
$$

The problem said to be multi-objective linear programming problem (MOLPP) if all the objective functions and constraint functions are linear, and all the variables are continuous variables.

\section{The New Technique for Solving MOLPP by Using Harmonic Mean}

Before solving MOLPP, and preface an algorithm to it, we will need to define Harmonic Mean.

\section{Harmonic Mean [4]}

Harmonic mean of a set of observations is defined as the reciprocal of the arithmetic average of the reciprocal of the given values. If $x_{1}, x_{2}, \cdots, x_{n}$ are $n$ observations, $H M=\frac{n}{\sum_{i=1}^{n}\left(\frac{1}{x_{i}}\right)}$

\section{Multi-Objective Functions Formulation}

Suppose we optimize (maximize or minimize) all the objective functions individually in (2.1), (2.2) and (2.3) and obtain the values as follows. 


$$
\left.\begin{array}{l}
\text { Max. } f_{1}=\Psi_{1} \\
\text { Max. } f_{2}=\Psi_{2} \\
\quad \vdots \\
\text { Max. } f_{r}=\Psi_{r} \\
\text { Min. } f_{r+1}=\Psi_{r+1} \\
\quad \vdots \\
\text { Min. } f_{s}=\Psi_{s}
\end{array}\right\}
$$

where $\Psi_{i},(i=1,2, \cdots, s)$ are the values of objective functions.

We require the common set of decision variable to be the best compromising optimal solution [5]. Here we can determine the common set of decision variables from the following combined objective function.

Formulate the multi-objective linear programming problem given in (1.1) can be translated by our technique to:

$$
\text { Max. } F=\sum_{k=1}^{r}\left(\operatorname{Max} . f_{k}\right) / H m_{1}-\sum_{k=r+1}^{s}\left(\operatorname{Min} . f_{k}\right) / H m_{2}
$$

where

$$
H m_{1}=r /\left(\sum_{i=1}^{r} 1 / \Psi_{i}\right), H m_{2}=(s-r) /\left(\sum_{i=r+1}^{s} 1 / \Psi_{i}\right)
$$

And $\Psi_{i} \neq 0 ;(i=1,2, \cdots, s)$ subject to the same constraints (1.2), (1.3) and the optimum value of the functions $\Psi_{k}(k=1,2, \cdots, s)$ may be positive or negative, $H m_{1}$ the value of harmonic mean of maximized $\Psi_{k}(k=1,2, \cdots, r)$ and $\mathrm{Hm}_{2}$ the value of harmonic mean of minimized $\Psi_{k}(k=r+1, \cdots, s) . s-r \geq 2$, if $s-r=1$ then the combined formula (3.2) becomes.

$$
\text { Max. } F=\sum_{k=1}^{r}\left(\text { Max. } f_{k}\right) / H m_{1}-\left(\text { Min. } f_{r+1}\right) /\left|\Psi_{r+1}\right|
$$

If $s-r=0$ then the function Max. $F=\sum_{k=1}^{r}\left(\right.$ Max. $\left.f_{k}\right) / H m_{1}$. We can solve this (MOLPP) by Chandra Sen's approach [1]-[3] by using mean and median and algorithms in above researches for solving MOLPP as explained in [1]-[3].

\subsection{Algorithm}

This algorithm is to obtain the optimal solution for the MOLPP defined previously can be summarized as follows.

Step 1: Assign arbitrary values to each of the individual objective functions that to be maximized and minimized.

Step 2: Solve the first objective function \{Max. $f_{1}$ subject to constraints (1.2) and (1.3)\} by simplex method.

Step 3: Check the feasibility of the solution in step 2, if it is feasible then go to step 4, otherwise, use dual simplex method to remove infeasibility.

Step 4: Assign a name to the optimum value of the first objective function f1 say $\Psi_{1}$

Step 5: Repeat step 2, for $i=1, \cdots, r$ for the $k$ th objective function, for all $i=i+1, \cdots, s$

Step 6: Determine Harmonic Mean $\mathrm{Hm} 1$ for $\Psi_{i}, i=1, \cdots, r$ and $\mathrm{Hm}_{2}$ for $i=r+1, \cdots, s$

Step 7: Optimize the combined objective function Max. $F=\sum_{k=1}^{r}\left(\operatorname{Max} . f_{k}\right) / H m_{1}-\sum_{k=r+1}^{s}\left(\operatorname{Min} . f_{k}\right) / H m_{2}$ under the same constraints (1.2) and (1.3) by repeating Steps 2-4.

\subsection{Used Notation}

The following notations were used in our algorithm:

$$
\begin{aligned}
& H A_{i}=\left|\Psi A_{i}\right|, \quad \forall i=1,2, \cdots, r \\
& H L_{i}=\left|\Psi L_{i}\right|, \quad \forall i=r+1, \cdots, s
\end{aligned}
$$


where $\Psi A_{i}=$ The value of objective function which is to be maximized, and

$\Psi L_{i}=$ The value of objective function which is to be minimized.

$H m_{1}=$ The value of Harmonic mean of maximized $\left(H m_{1}=r /\left(\sum_{i=1}^{r} 1 / \Psi_{i}\right)\right)$.

$\mathrm{Hm}_{2}=$ The value of Harmonic mean of minimized $\left(H m_{2}=(s-r) /\left(\sum_{i=r+1}^{s} 1 / \Psi_{i}\right)\right)$.

$S L=\sum_{i=1}^{r} \operatorname{Max} . f_{i} ; \quad S S=\sum_{i=r+1}^{s} \operatorname{Min} . f_{i}$

$\operatorname{Max} . F=S L / H m_{1}-S S / H m_{2}$

\subsection{Numerical Examples}

Ex. (1)

$$
\left.\begin{array}{l}
\text { Max. } f_{1}=x_{1}+2 x_{2} \\
\text { Max. } f_{2}=3 x_{1} \\
\text { Min. } f_{3}=-2 x_{1}-3 x_{2} \\
\text { Min. } f_{4}=-x_{2}
\end{array}\right\}
$$

s.to:

$$
\left.\begin{array}{l}
6 x_{1}+8 x_{2} \leq 48 \\
x_{1}+x_{2} \geq 3 \\
x_{1} \leq 4 \\
x_{2} \leq 3 \\
x_{1}, x_{2} \geq 0
\end{array}\right\}
$$

Solution:

After finding the value of each of individual objective function by simplex method the results as below in (Table 1): $\left\{\Psi_{1}=10, \Psi_{2}=4, \Psi_{3}=-17, \Psi_{4}=-3\right\}$ by using Harmonic Mean technique (3.3) we get $H m_{1}=80 / 14$ and $\mathrm{Hm}_{2}=51 / 10$

After that using equation (3.2) for transform we get:

$$
\left.\begin{array}{l}
\text { Max. } F=0.7421 x_{1}+1.1343 x_{2} \\
\text { Subject to given constraints }(5.2):
\end{array}\right\}
$$

Solving (4.3) by simplex method we get:

$$
\text { Max. } F=6.3715 \quad x_{1}=4, \quad x_{2}=3
$$

Solve (4.1) by:

1. Using Chandra Sen's approach, [1]: after convert the MOLPP to the single objective problem we get Max. $F=0.4676 x_{1}+0.7098 x_{2}$ subject to the same constraints (4.2) by simplex method it is optimal solution Max. $F=3.9999 x_{1}=4, x_{2}=3$.

Table 1. Results of example (1).

\begin{tabular}{ccccccc}
\hline$I$ & $\Psi_{i}$ & $X_{i}$ & $H A_{1}$ & $H L_{i}$ & $H m_{1}$ & $H m_{2}$ \\
\hline 1 & 10 & $(4,3)$ & 10 & & & \\
2 & 4 & $(4,3)$ & 4 & & & \\
3 & -17 & $(4,3)$ & & 3 & $51 / 14$ \\
4 & -3 & $(4,3)$ & & & 510 \\
\hline
\end{tabular}


2. Using modified approach, [2]:

A-using Mean: after convert the MOLPP to the single objective problem we get Max. $F=0.4857 x_{1}+0.7857 x_{2}$ subject to the same constraints (4.2) by simplex method it is optimal solution Max.F $=4.2999 x_{1}=4, x_{2}=3$

B-using Median: after convert the MOLPP to the single objective problem we get

Max. $F=0.4857 x_{1}+0.7857 x_{2}$ subject to the same constraints (4.2) by simplex method it is optimal solution Max. $F=4.2999 x_{1}=4, x_{2}=3$.

Ex. (2)

$$
\left.\begin{array}{l}
\text { Max. } f_{1}=x_{1} \\
\text { Max. } f_{2}=2+x_{1}+2 x_{2} \\
\text { Max. } f_{3}=3+x_{2} \\
\text { Min. } f_{4}=-3 x_{2} \\
\text { Min. } f_{5}=-x_{1}-3 x_{2}
\end{array}\right\}
$$

Subject to:

$$
\left.\begin{array}{l}
2 x_{1}+3 x_{2} \leq 6 \\
x_{1} \leq 4 \\
x_{1}+2 x_{2} \leq 2 \\
x_{1}, x_{2} \geq 0
\end{array}\right\}
$$

Solution:

After finding the value of each of individual objective function by simplex method the results as below in (Table 2): $\left\{\Psi_{1}=2, \Psi_{2}=4, \Psi_{3}=4, \Psi_{4}=-3\right.$ and $\left.\Psi_{5}=-3\right\}$ by using Harmonic Mean technique (3.3) we get $H m_{1}=3$ and $\mathrm{Hm}_{2}=3$.

After that using equation (3.2) for transform we get:-

$$
\left.\begin{array}{l}
\text { Max. } F=5+3.5 x_{1}+12 x_{2} \\
\text { Subject to given constraints }(6.2):
\end{array}\right\}
$$

Solving (5.3) by simplex method we get:

$$
\text { Max. } F=4.6 \quad x_{1}=0, x_{2}=1
$$

Solve (5.1) by:

1. using Chandra Sen approach, [1]: after convert the MOLPP to the single objective problem we get Max. $F=1.25+1.08333 x_{1}+2.75 x_{2}$ subject to the same constraints (5.2) by simplex method it is optimal solution Max. $F=4 x_{1}=0, x_{2}=1$.

2. using modified approach, [2]:

A-using Mean: after convert the MOLPP to the single objective problem we get

Max. $F=1.5+0.93333 x_{1}+2.9 x_{2}$ subject to the same constraints (5.2) by simplex method it is optimal solution Max. $F=4.4 \quad x_{1}=0, x_{2}=1$.

B-using Median: after convert the MOLPP to the single objective problem we get

Table 2. Results of example (2).

\begin{tabular}{ccccccc}
\hline$I$ & $\Psi_{i}$ & $X_{i}$ & $H A_{i}$ & $H L_{i}$ & $H m_{1}$ & \\
\hline 1 & 2 & $(2,0)$ & 2 & & \\
2 & 4 & $(0,1)$ & 4 & & \\
3 & 4 & $(0,1)$ & 4 & 3 & 3 \\
4 & -3 & $(0,1)$ & & 3 & \\
5 & -3 & $(0,1)$ & & & \\
\hline
\end{tabular}


Table 3. Comparison between results obtained by different numerical approaches.

\begin{tabular}{ccccc}
\hline \multirow{2}{*}{ Examples } & Chandra Sen's approach & \multicolumn{2}{c}{ Modified approach } & \multicolumn{2}{c}{$\begin{array}{c}\text { New approach } \\
\text { using harmonic } \\
\text { mean }\end{array}$} \\
\cline { 3 - 5 } & Max. $F=3.9999 x_{1}=4, x_{2}=3$ & Max. $F=4.2999 x_{1}=4, x_{2}=3$ & Max. $F=4.2999 x_{1}=4, x_{2}=3$ & Max. $F=6.3715 x_{1}=4, x_{2}=3$ \\
Example (2) & Max. $F=4 x_{1}=0, x_{2}=1$ & Max. $F=4.4 x_{1}=0, x_{2}=1$ & Max. $F=4 x_{1}=0, x_{2}=1$ & Max. $F=4.6 x_{1}=0, x_{2}=1$ \\
\hline
\end{tabular}

Max. $F=1.25+0.83333 x_{1}+2.75 x_{2}$ subject to the same constraints (6.2) by simplex method it is optimal solution Max. $F=4 x_{1}=0, x_{2}=1$.

\section{Conclusions}

Our aim was to develop an approach for solving multi-objective programming problem (MOLPP) and to suggest a new algorithm to convert the MOLPP into a single LPP by using harmonic mean of the values of objective functions and its computer application by using programming mathematical language (Matlab Programming). Moreover, we used different methods to solve the problems, and applied our technique and the other methods to the same examples in order to compare the results.

From this comparison, we observed that our technique gave identical results with that obtained by the other methods, for this see Table 3. So we conclude that this method is better than other methods considered in solving MOLP problems.

\section{References}

[1] Chandra, S. (1983) A New Approach Objective Planning. The Indian Economic Journal, 30, 91-96.

[2] Sulaiman, N.A. and Sadiq Gulnar, W. (2006) Solving the Multi-Objective Programming Problem; Using Mean and Median Value. Ref. J of Comp \& Math's, 3.

[3] Abdul-Kadir, M.S. and Sulaiman, N.A. (1993) An Approach for Multi-Objective Fractional Programming Problem. Journal of College of Education, University of Salahaddin, 3, 1-5.

[4] Jothikumar, J. (2004) STATISTICS. 60 G.S.M Paper, p.100.

[5] Azapagic, A. and Clift, R. (1999) Life Cycle Assessment and Multi Objective Optimization. Journal of Cleaner Production, 7, 135-143. http://dx.doi.org/10.1016/S0959-6526(98)00051-1 